Military Technical College

Kobry Elkobbah,

Cairo, Egypt

April 19-21,2016

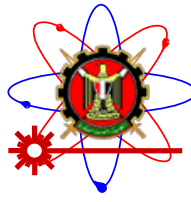

$8^{\text {th }}$ International Conference on Mathematics and Engineering Physics (ICMEP-8)

\title{
EM-2
}

\section{A New Method For Synchronization Of A Simple Chaotic systems}

\author{
Abdel Abdel-Azeem Mohamed and Samy H. Darwish \\ Faculty of Engineering, Pharos University, Alexandria, Egypt \\ Azima65@hotmail.com, and Salord1960@yahoo.com
}

\begin{abstract}
This paper presents a new algorithm for controlling a class of simple chaotic system that contain only one nonlinear term. Chaos synchronization using parametric controllers is generalized for typical simple chaotic systems based on largest conditional Lyapunov exponents. A more robust and rigorous definition can be given in terms of the Lyapunov exponents. When the Lyapunov exponents for system are all negative, the systems will synchronize. The Pecora -Carroll method is one of the methods for synchronizing chaotic systems. However, the method is restricted to select the driving signals or its configuration. The proposed method does not depend on the choice of the drive signal or its configuration; it depends only on the nonlinear coupling term which makes the largest conditional Lyapunov exponents of the response system negative. The comparison between the implementation of presented method and the Pecora -Carroll(PC) method is explained. Simulations are presented graphically to confirm the validity of the proposed method
\end{abstract}

Keywords: Chaotic system; Lyapunov exponents, synchronization, Stability

\section{1-Introduction}

Chaotic systems have been widely studied by many scientists and engineers from different viewpoints. Chaos is one of the most significant topics in nonlinear science, and has been intensively studying since the Lorenz system [1] was introduced. After many chaotic systems have been discovered and developed, scientists have focused on chaos control and chaos synchronization since 1990s. Chaos synchronization was discovered by Pecora and Carroll [2] and there has been great interest in its applications, such as secure communication, and system identification. Given a chaotic system considered as a master system, and another identical system considered as a slave system, the dynamical behaviors of them may be identical after a transient when the slave system is driven by a control input. There are many methods for controlling chaos such as feedback and synchronization techniques [1-4]. 
The synchronization of two chaotic dynamical system occurs when the trajectories of one the systems will converge to the other system at the same time or the two systems show the same behavior at the same time. Different methods for synchronization such as complete synchronization, phase synchronization, lag synchronization and generalized synchronizations have been presented [3-6].

To achieve synchronization, there are many methods for linking chaotic systems. Famous two methods are: the linear diffusive coupling, as initially suggested by Fujisaka and Yamada [8 and 9], and driving coupling, introduced by Pecora and Carroll[5-7]. There are another additive coupling which is offered by the open-plusclosed- loop method to control and synchronize chaotic systems developed by Jackson and Grosu[10-12] .

Concerning stability, there are two criteria which are most commonly used for stability of synchronized chaotic motions, these two criteria are the Lyapunov function criterion [12,13,17] and the conditional Lyapunov exponents [13-15] . In many practical cases, Lyapunov functions cannot be found, even for systems that possess a stable manifold of synchronized motions for a broad range of parameters of coupled systems, and/or the coupling itself. In contrast with Lyapunov functions, the analysis of conditional Lyapunov exponents $[13,14,15]$ is quite straightforward and can be easily employed, even for rather complicated systems. The presence of synchronization can occur if the conditional Lyapunov exponents of the response system are negative.

Lyapunov exponents are commonly used for chaos identification in nonlinear dynamical systems by showing the average rate of growing or shrinking of asmall volume of initial conditions. These exponents provide a quantitative measure for the sensitivity of the nonlinear system to the change of initial conditions. Also Lyapunov exponents demonstrate the chaotic behavior of the system [12, 13], and there are several methods for numerical calculation of Lyapunov exponents $[14,15]$.

The control of typical chaotic system by synchronization is the aim of this work. Thus a method suggested by forcing the largest Lyapunov exponent of the response system to be negative. This is achieved by combining the open -loop-closed -loop method and Routh -Hurwitz criteria [16, 17].

\section{Synchronization}

This section reviews the Pecora and Carroll method and introduces the proposed method for synchronization of chaotic system.

\subsection{Pecora and Carroll method.}

This method is a widely used approach synchronization problem [5-7]. Consider an ndimensional autonomous system governed by the equation

$$
\frac{d x}{d t}=f(x) \quad x=\left(x_{1}, x_{2}, \ldots, x_{n}\right)^{T}
$$

the system into two parts in an arbitrary way, i.e. dividing the state vector into $\mathrm{X}=[\mathrm{xD}, \mathrm{xR}]^{\mathrm{T}}$. The $\mathrm{D}$ is referred to as the driving subsystem, and $\mathrm{R}$ is referred to as the response subsystem respectively, then 


$$
\begin{aligned}
& x_{D}=g\left(x_{D}, x_{R}\right), \\
& x_{R}=h\left(x_{D}, x_{R}\right),
\end{aligned}
$$

where,

$$
\begin{aligned}
& x_{D}=\left(x_{1}, x_{2}, \ldots, x_{m}\right)^{T}, \\
& x_{R}=\left(x_{m+1}, \ldots x_{n}\right)^{T}, \\
& g=\left[f_{1}(x), \ldots, f_{m}(x)\right]^{T}, \\
& h=\left[f_{m+1}(x), \ldots, f_{n}(x)\right]^{T} .
\end{aligned}
$$

Pecora and Carroll suggested building an identical copy of the response subsystem and driving it with the $X_{D}$ variable coming from the original system. In such a method, the following compound system of equation can be found

$$
\begin{aligned}
& \boldsymbol{x}_{D}=\boldsymbol{g}\left(\boldsymbol{x}_{D}, \boldsymbol{x}_{R}\right), \text { (m-dimensional)-drive } \\
& \boldsymbol{x}_{R}=\boldsymbol{h}\left(\boldsymbol{x}_{D}, \boldsymbol{x}_{R}\right), \text { (k-dimensional)- drive } \\
& \boldsymbol{x}_{D}=\boldsymbol{h}\left(\boldsymbol{x}_{D}, \boldsymbol{x}_{R}^{\prime}\right), \text { (k-dimensional)-response }
\end{aligned}
$$

\subsubsection{Theorem 1}

The subsystems $X_{R}$ and $X_{R}^{\prime}$ will synchronize only if the conditional Lyapunov exponents are all negative [5].

Under the right conditions, the $\boldsymbol{x}_{\boldsymbol{R}}^{\prime}$ variable will converge asymptotically to the $\boldsymbol{X}_{\boldsymbol{R}}$ variable and continue to remain in step with instantaneous value of $\boldsymbol{X}_{\boldsymbol{R}}(\boldsymbol{t})$. Here the drive or master system controls the response or slave system through $\boldsymbol{X}_{\boldsymbol{D}}$ component. If all conditional Lyapunov exponents of the response system are negative then the synchronization occurs. Otherwise the synchronization does not occur if at least one of the conditional Lyapunov exponents is positive.

\subsubsection{Lyapunov exponents}

The idea of synchronizing two identical chaotic systems that start from different initial conditions can occur when the largest Lyapunov exponents for the subsystem to be synchronized are all negative. The Lyapunov exponent measures the growth of small perturbation of the difference between two systems. The next paragraph introduces briefly how to calculate the Largest Lyapunov exponents of the whole system after synchronization [13-15].

It is known that under suitable coupling one system will follow another system if its largest conditional Lyapunov exponent is negative. The largest Lyapunov exponents 
of the whole system can calculate the distance between the subsystem and the original system which is

$\sqrt{\sum_{i=1}^{2 N} \partial^{2}} x_{0}(i) . \quad$ Where $\mathrm{N}$ is

With the evaluation of time, the distance will be expanded along the largest eigenvalues directions, so the largest Lyapunov exponents can be obtained as follows

$$
\lambda=\lim _{t \rightarrow \infty} \frac{1}{t} \ln \sqrt{\sum_{i=1}^{2 N} \partial^{2} x_{0}(i)} / \sqrt{\sum_{i=1}^{2 N} \partial^{2} x_{t}(i)}
$$

\subsection{The proposed method of Synchronizion}

Some undesirable characteristics of the Pecora and Carroll method are the existence of the positive conditional Lyapunov exponent and the dependence on the configuration of the drive signal. The proposed method avoids this problem by choosing the values of system parameters that make the largest Lyapunov exponents negative using the concept of Routh- Hurwitz criteria and the dependence on the chosen of drive signal are discarded.

The proposed method depends upon the method of Open- Plus- Closed- Loop for control of dynamical systems (OPCL) [10-12].which is summarized as follows

Consider the dynamical system is given by:

$$
\frac{d x}{d t}=F(x), \quad\left(x \in R^{n}\right)
$$

if one wants to entrain the solution of a dynamical systems to some goal behavior , $g(t)$, using linear feedback, in order to obtain $\lim _{t \rightarrow \infty}(x(t)-g(t))=0 \quad$ the dynamics is of the form

$$
\frac{d x}{d t}=F(x, t)+D(t, x, g)
$$

where, $D(t, x, g)$ is the drive term and is given by:

$$
D(t, x, g)=\frac{d g}{d t}-F(g, t)+\left(A-\left.\frac{\partial F}{\partial x}\right|_{x=g}\right)(x-g) \text {, }
$$

Where $\mathrm{A}$ is a constant matrix with eigenvalues having negative real part, $\mathrm{D}$ is some suitable matrix in this case $\mathrm{g}(\mathrm{t})$ has to be restricted to some solution of

$$
\frac{d g}{d t}=F(g, t)
$$

The idea of the proposed method is replacing the parameter $\mathrm{P}$ in place of the nonlinear term in the constant matrix of the Jacobian of the master system and choosing the value of the parameter $\mathrm{P}$ that makes the eigenvalues having negative real part and also satisfying the Routh - Hurwitz criteria for stability then derive the slave system by the drive term as follows

$$
\left(\mathrm{A}-\left.\frac{\partial F}{\partial x}\right|_{x=y}\right)(\mathrm{x}-\mathrm{y})
$$

consider the dynamical system given by: 


$$
\frac{d x}{d t}=F(x, t)
$$

Then the slave system will be:

$$
\frac{d y}{d t}=F(y, t)+\left(\mathrm{A}-\left.\frac{\partial F}{\partial x}\right|_{x=y}\right)(\mathrm{x}-\mathrm{y})
$$

Where, $A$ is a constant matrix with eigenvalues with negative real parts. Then $x(t)$ converges to $\mathrm{y}(\mathrm{t})$ for any $\| \mathrm{x}(0)$ - $\mathrm{y}(0) \|$ small enough.

The proposed method is based on the analysis of the characteristic polynomial coefficients of the matrix A. Let the characteristic polynomial of A be denoted by:

$$
A(s)=a_{0} s_{n}+a_{1} s_{n-1}+\ldots+a_{n}=0, a_{0}>
$$

As presented in [10], it is possible to derive necessary and sufficient conditions for the existence of eigenvalues with negative real part by applying Routh- Hurwitz conditions. By applying these conditions, it is possible to determine the numerical interval of parameter that insure the eigenvalues in the left hand plane

\subsubsection{The proposed method}

The algorithm of the proposed method is given by

1-Find the Jacobian matrix of the master system $J=\frac{\partial F}{\partial x}$

2-Find the matrix A by replacing the element containing the variable in the matrix $J$ by the parameter $\mathrm{P}$ (coupling term)

3-Find the characteristic equation of the matrix A

4-Applying the Routh- Hurwitz criteria to find the range values of $\mathrm{P}$ and substitute it in the matrix $\mathrm{A}$

5-Find the drive term $\left(\mathrm{A}-\left.\frac{\partial F}{\partial x}\right|_{x=y}\right)(\mathrm{x}-\mathrm{y})$

7-Create the synchronization algorithm according to the range of parameter $\mathrm{P}$ as follows

$$
\begin{aligned}
& \text { The master system } \quad \frac{d x}{d t}=F(x, t) \\
& \text { The slave system } \frac{d y}{d t}=F(y, t)+\left(\mathrm{A}-\left.\frac{\partial F}{\partial x}\right|_{x=y}\right)(\mathrm{x}-\mathrm{y})
\end{aligned}
$$

8-Test for the synchronization ( calculate the largest Lyapunov exponents) the algorithm is coded in $\mathrm{C}++$

\section{$\underline{3 \text { Case Study }}$}

The following system will be analyzed using the suggested method and the PecoraCarroll method 


\subsection{Simple chaotic system 1}

Consider the following system [14] is described by

$$
\begin{aligned}
& \frac{d x}{d t}=y+z \\
& \frac{d y}{d t}=-x+0.5 y \\
& \frac{d z}{d t}=x^{2}-z
\end{aligned}
$$

The system 1 has only six terms, a single quadratic nonlinearity $\left(\mathrm{x}^{2}\right)$, and two critical points $(-2,-4,4)$ and $(0,0,0)$. The largest Lyapunov exponent of the system is equal to $(0.1342)$ and the chaotic behavior of the system is shown in figure (1)

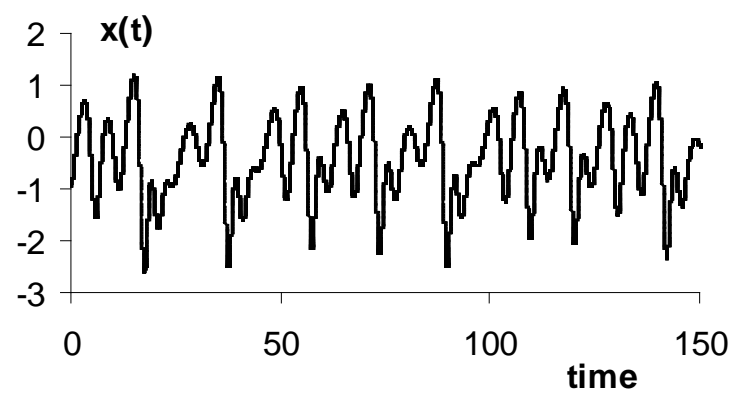

Figure 1a- the time response of $\mathrm{x}(\mathrm{t})$ of system 1

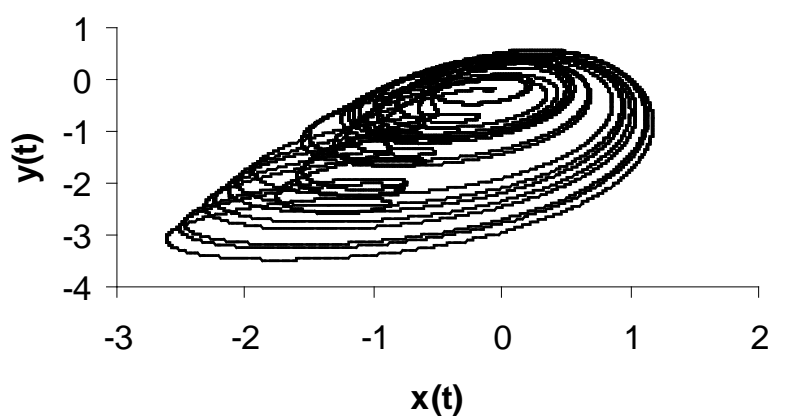

Figure $1 \mathrm{~b}$ - the phase plane plot of $\mathrm{x}(\mathrm{t})$ versus $\mathrm{y}(\mathrm{t})$ of system

By applying the concept of The Pecora and Carroll to the system using y- drive configuration one can get 


$$
\begin{aligned}
& \frac{d x}{d t}=y+z \\
& \frac{d y}{d t}=-x+0.5 * y \\
& \frac{d z}{d t}=x^{2}-z \\
& \frac{d x_{1}}{d t}=y+z_{1} \\
& \frac{d z_{1}}{d t}=x_{1}^{2}-z_{1}
\end{aligned}
$$

The conditional Lyapunov exponents of the subsystem are $(-0.17,-.84)$ i.e. the synchronization occurs. Also the largest Lyapunov exponents of the whole system is 0.102 and the system after synchronization is chaotic. Figure (2) shows the synchronization of the chaotic system in case of $y$ - drive

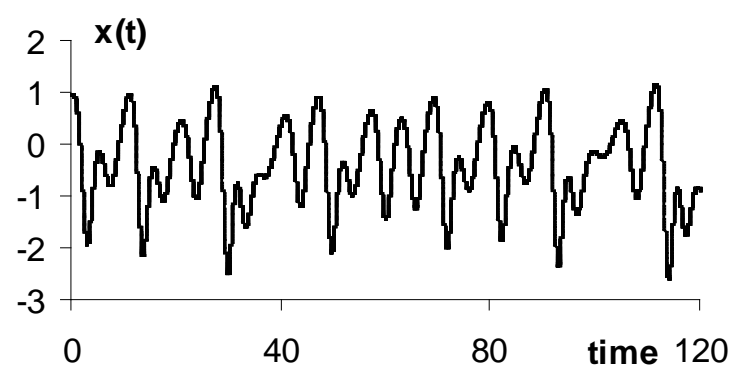

Figure 2a- The time response of $\mathrm{x}(\mathrm{t})$ of the drive system using PC method

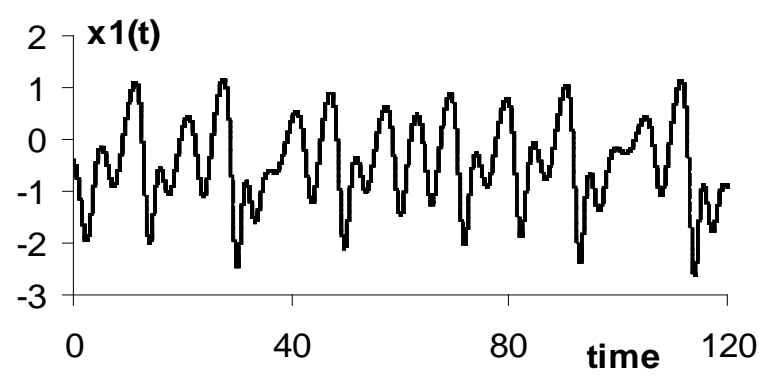

Figure 2b- The time response of $\mathrm{x} 1(\mathrm{t})$ of the response system using PC method 


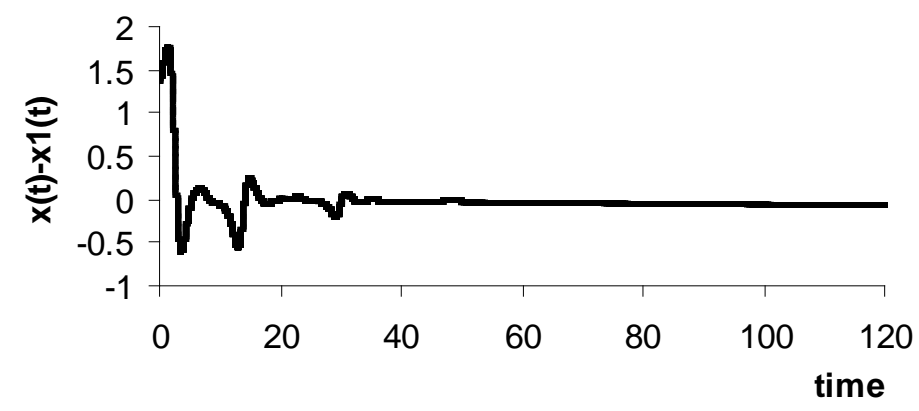

Figure 2c- The error of synchronization between $\mathrm{x}(\mathrm{t}), \mathrm{x} 1(\mathrm{t})$ using PC method

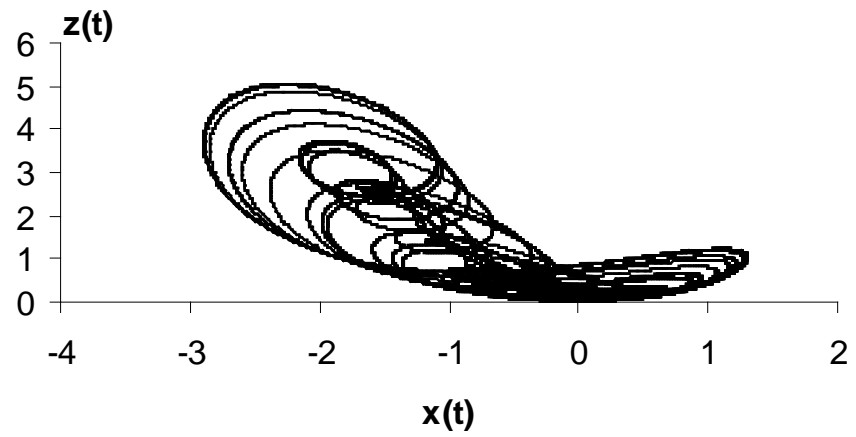

Figure $2 \mathrm{~d}$ - The phase plane $(\mathrm{x}(\mathrm{t})$ versus $\mathrm{z}(\mathrm{t}))$ of whole system after synchronization using PC method ( non -oscillating behavior)

In the case of synchronization by Pecora and Carroll method the synchronization does not occur and it depends on the configuration for driving signal. For example, in the $\mathrm{x}(\mathrm{t})$ configurations the subsystems does not synchronize, the conditional Lyapunov exponent positive, the Conditional Lyapunov exponent for drive-response systems of systems is shown in table 2 .

\begin{tabular}{|c|c|c|c|}
\hline system & Drive signal & $\begin{array}{l}\text { Response } \\
\text { system }\end{array}$ & $\begin{array}{l}\text { Conditional } \\
\text { Lyapunov exponents }\end{array}$ \\
\hline \multirow[t]{3}{*}{ System 2} & $\mathrm{x}$ & $(\mathrm{y}, \mathrm{z})$ & $\begin{array}{l}\text { +ve } \\
\text { ( no synchronization }\end{array}$ \\
\hline & $\mathrm{y}$ & $(\mathrm{x}, \mathrm{z})$ & $\begin{array}{l}\text {-ve(non- } \\
\text { synchronization behavior }\end{array}$ \\
\hline & $\mathrm{Z}$ & $(\mathrm{x}, \mathrm{y})$ & $\begin{array}{l}\text { +ve } \\
\text { ( no synchronization) }\end{array}$ \\
\hline
\end{tabular}

Table 1- Conditional Lyapunov exponent for system1 for different drive signals using PC method 
The results are in a good agreement with other results that published in [16]. To avoid this problem, - the new algorithm can be used for synchronization, the algorithm selects the drive signal directly from the nonlinear part.

By applying the method outlined in section (3) then the master system is given by equation (14) the Jacobian matrix is given by

$$
J=\left[\begin{array}{lcc}
0 & 1 & 1 \\
-1 & 0.5 & 0 \\
2 p & 0 & -1
\end{array}\right]
$$

The coupling terms comes from the matrix

$$
A=\left[\begin{array}{lcc}
0 & 1 & 1 \\
-1 & 0.5 & 0 \\
2 p & 0 & -1
\end{array}\right]
$$

Where $P$ is a parameter that has to be chosen to make the eigenvalues of $\mathrm{A}$ having negative real parts. The characteristics equation is given by:

$$
\lambda^{3}+.5 \lambda^{2}+.5 \lambda+1-2 p \lambda+1.0 p=0
$$

With , a1 $=0.5$, a $2=0.5-2 \mathrm{P} \quad$ and $\mathrm{a} 3=1+\mathrm{P}$

The Routh - Hurwitz conditions are a1 $>0, \mathrm{a} 3>0 \quad$ and $\mathrm{a} 1 \mathrm{a} 2-\mathrm{a} 3>0$

Given the condition $\quad-2>\mathrm{P}>-0.75$, then the slave system is given by

$$
\begin{aligned}
& \frac{d x_{1}}{d t}=y_{1}+z_{1} \\
& \frac{d y_{1}}{d t}=-x_{1}+0.5 y_{1} \\
& \frac{d z_{1}}{d t}=\left(x_{1}\right) 2-z_{1}+(p-2 x)\left(x_{1}-x\right)
\end{aligned}
$$

Figure (3) shows the synchronization of the chaotic system using the proposed method 


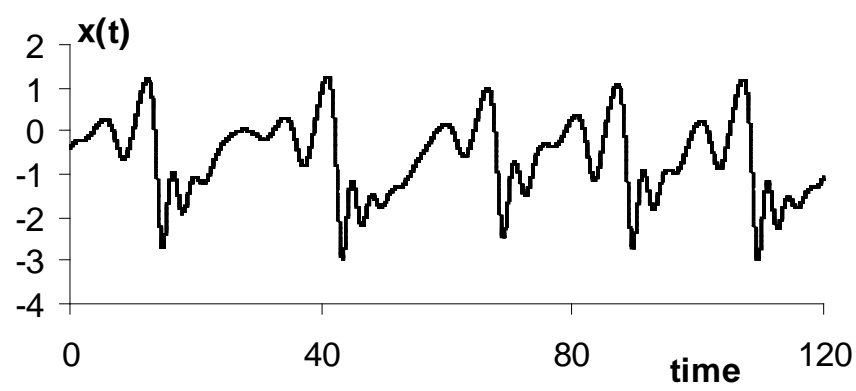

Figure 3a- The time response of $\mathrm{x}(\mathrm{t})$ of the drive system using the proposed method

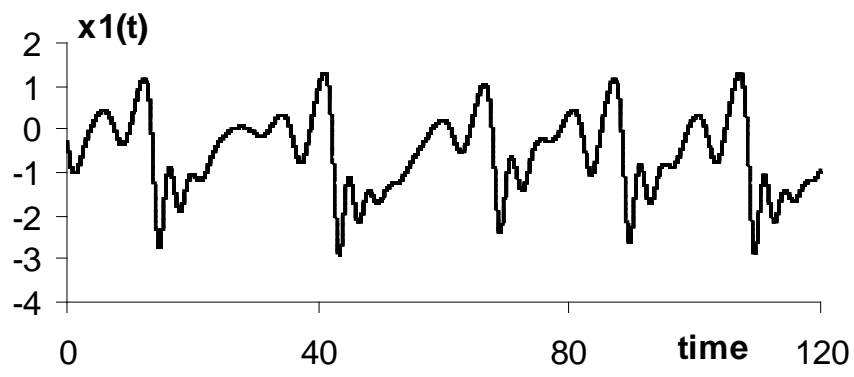

Figure 3b- The time response of $\mathrm{x} 1(\mathrm{t})$ of the response system 1 using the proposed method

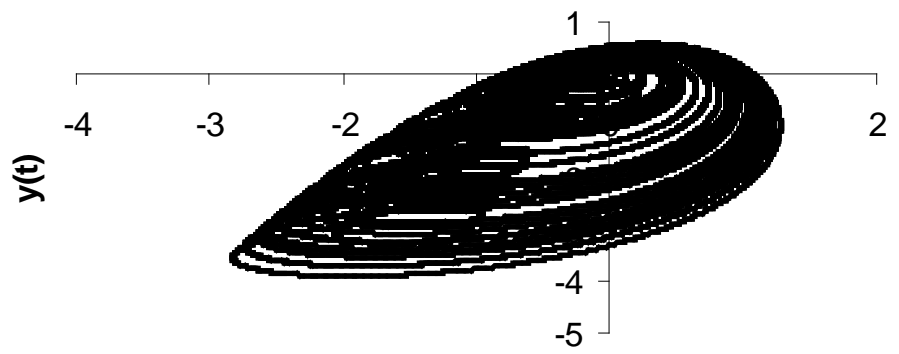

$x(t)$

Figure 3c- The phase plane plot of $x(t)$ vs. $y(t)$ the system after synchronization using the proposed method 


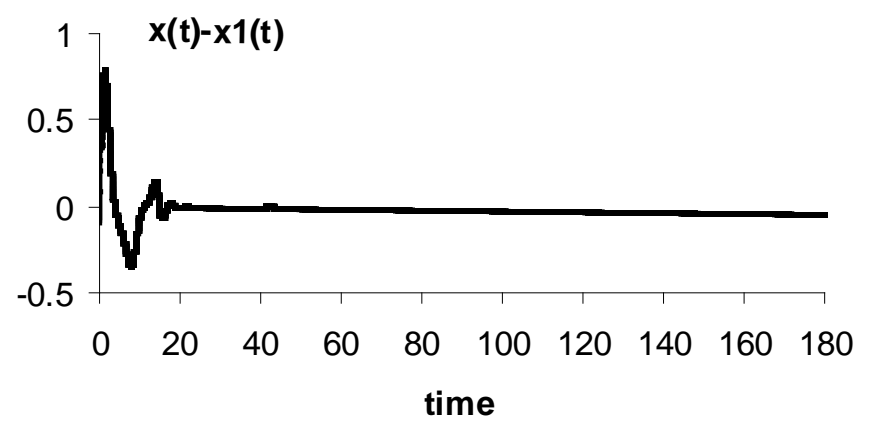

Figure 3d- The error of synchronization between $\mathrm{x}(\mathrm{t})$, $\mathrm{x} 1(\mathrm{t})$ using the proposed method

From the previous figures one can notice that synchronization process is archiving without selecting the type of configurations of the drive signal. Also, the error of synchronization in the proposed method is smaller than the method of Pecora -Carroll and the new method does not depend on the configuration of the drive signal also the new method detect the type of synchronization behavior.

Another proof to validate the proposed method by illustrating that in some system the method of PC is not applicable and synchronization not occur with different drive signals but the new algorithm avoids this problem.

The system 2 is described in $[14,16]$ as:

$$
\begin{aligned}
& \frac{d x}{d t}=a-y \\
& \frac{d y}{d t}=b+z \\
& \frac{d z}{d t}=x \quad y-z
\end{aligned}
$$

This system has six terms, a single nonlinearity (x y), and two parameters a and b. Letting $a=0.9$, and $b=0.4$, these values lead to the chaotic behavior with largest Lyapunov exponent (0.064). Its time response and phase plane plots are shown in Figure (4) 


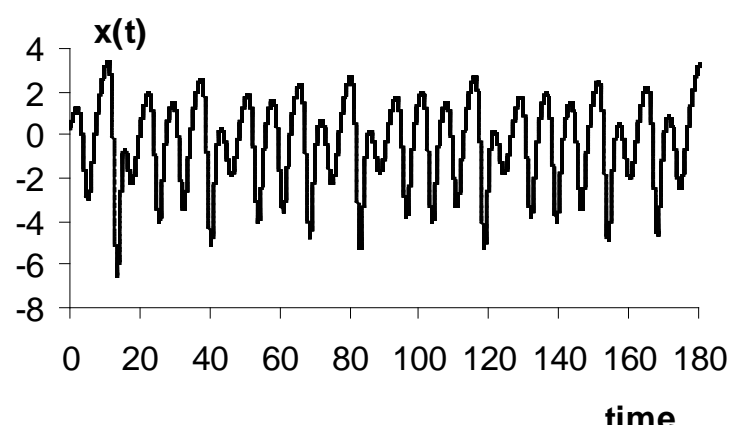

Figure 4a- The time response of $\mathrm{x}(\mathrm{t})$ of the system 2 described by equation (23)

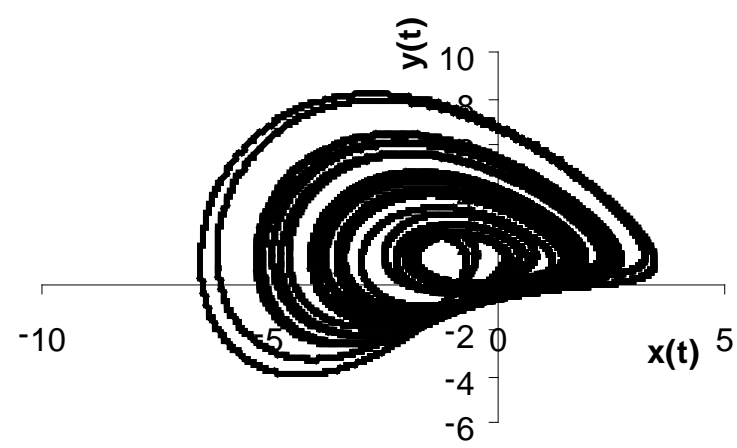

Figure $4 \mathrm{~b}$ - The phase plane $(\mathrm{x}(\mathrm{t})$ versus $\mathrm{y}(\mathrm{t}))$ of the system 2 described by equation (23)

By applying the concept of the Pecora and Carroll to the system using $\mathrm{x}$ - drive configuration one can get

$$
\begin{aligned}
& \frac{d x}{d t}=a-y \\
& \frac{d y}{d t}=b+z \\
& \frac{d z}{d t}=x \quad y-z \\
& \frac{d y_{1}}{d t}=b+z_{1} \\
& \frac{d z_{1}}{d t}=x y_{1}-z_{1}
\end{aligned}
$$

The conditional Lyapunov exponents of the subsystem are $(0.052,0.04)$, i.e. the synchronization will not occur. Also the largest Lyapunov exponent of the whole 
system is positive and the system is not synchronized. Also the same result is appears in the case of $\mathrm{y}$ and $\mathrm{z}$ as a drive signals and the system is not synchronized by the method of Pecora- Carroll. The Conditional Lyapunov exponents for the driveresponse systems of systems are shown in table 2 .

\begin{tabular}{|l||l||l||l|l||}
\hline \hline system & Drive signal & $\begin{array}{l}\text { Response } \\
\text { system }\end{array}$ & $\begin{array}{l}\text { Conditional } \\
\text { Lyapunov } \\
\text { exponents }\end{array}$ & Synchronization \\
\hline \hline $\begin{array}{l}\text { System of } \\
\text { equation } \\
(23)\end{array}$ & $\mathrm{x}$ & $(\mathrm{y}, \mathrm{z})$ & $+\mathrm{ve}$ & $\begin{array}{l}(\text { no } \\
\text { synchronization no }\end{array}$ \\
\cline { 2 - 6 } & $\mathrm{y}$ & $(\mathrm{x}, \mathrm{z})$ & $+\mathrm{ve}$ & $\begin{array}{l}(\text { no } \\
\text { synchronization })\end{array}$ \\
\cline { 2 - 5 } & $\mathrm{z}$ & $\mathrm{x}, \mathrm{y})$ & $+\mathrm{ve}$ & $\begin{array}{l}\text { ( } \\
\text { synchronization })\end{array}$ \\
\hline
\end{tabular}

Table 2- Conditional Lyapunov exponents for the system 2 for different drive signals using PC method

The synchronization not occur for different drive signals using PC method . To avoid this problems the new algorithm can be used for synchronization, the algorithm selects the drive signal directly from the nonlinear part.

By applying the method outlined in section (2) then the master system is given by equation (17) the Jacobian matrix is given by

$$
J=\left[\begin{array}{ccc}
0 & -1 & 1 \\
0 & 0 & 1 \\
y & x & -1
\end{array}\right]
$$

The coupling terms comes from the arbitrary matrix

$$
A=\left[\begin{array}{ccc}
0 & -1 & 1 \\
0 & 0 & 1 \\
1 & p & -1
\end{array}\right]
$$

Where, $\mathrm{P}$ is a parameter that has to be chosen to make the eigenvalues of A containing negative real parts. The characteristics equation is

$$
\lambda 3+\lambda 2-\mathrm{p} \lambda+1=0
$$

with a1 $=1$, a2 $=-\mathrm{P} \quad$ and $\mathrm{a} 3=1$ 
The Routh - Hurwitz conditions are a1 $>0$, a3 $>0$ and $\mathrm{a} 1 \mathrm{a} 2-\mathrm{a} 3>0$

Given the condition $\mathrm{P}<-1$, then the slave system is given by

$$
\begin{aligned}
& \frac{d x_{1}}{d t}=0.9-y_{1} \\
& \frac{d y_{1}}{d t}=0.4+z \\
& \frac{d z_{1}}{d t}=x_{1} y_{1}-z_{1}+(1-y)\left(x_{1}-x\right)+(p-x)\left(y_{1}-y\right)
\end{aligned}
$$

Figure (5) shows the synchronization of the chaotic system using the proposed method

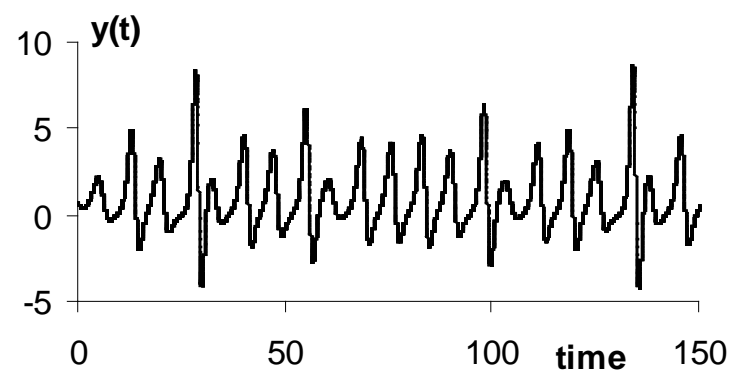

Figure 5a- The time response $\mathrm{y}(\mathrm{t})$ of the drive system 2 using the proposed method

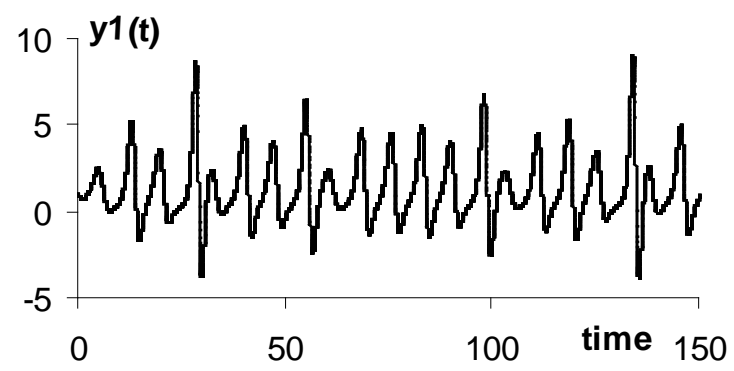

Figure 5b- The time response $\mathrm{y} 1 \mathrm{t}$ ) of the response system using the proposed method 


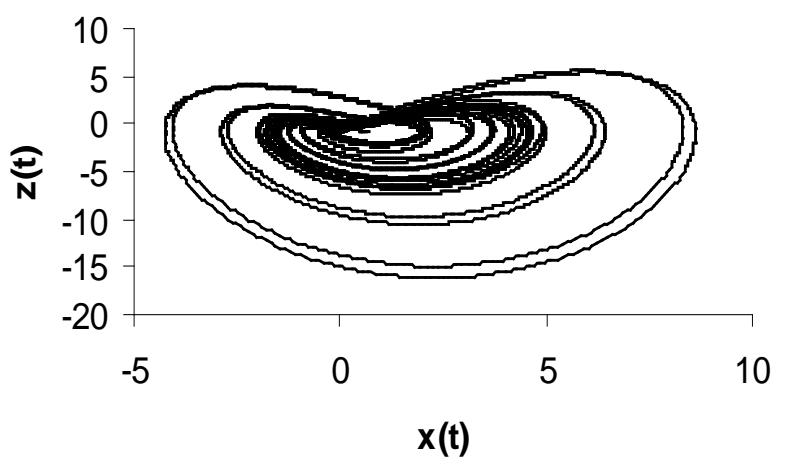

Figure 5c- The phase plane plot of $\mathrm{x}(\mathrm{t}) \mathrm{vs}$. $\mathrm{z}(\mathrm{t})$ the system after synchronization using the proposed method (the largest Lyapunov exponent of the whole system (0.023) and the system is in case of chaotic synchronization )

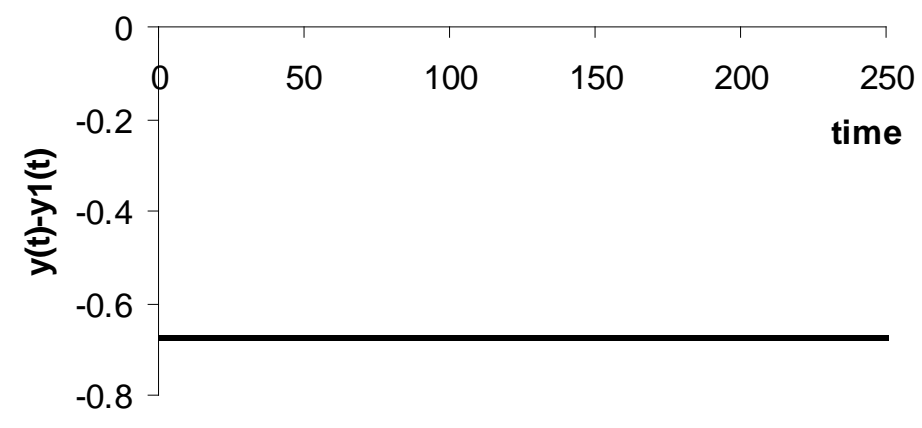

Figure 5d- the error of synchronization between $\mathrm{y}(\mathrm{t}), \mathrm{y} 1(\mathrm{t})$ using the proposed method (constant shift marginal synchronization (-0.692))

From the previous figures it can be noted that the synchronization process is achieved without selecting the type of configurations of the drive signal, and the system is synchronized but with using the method of Pecora and Carroll the synchronization does not occur. The proposed algorithm is applicable in some regimes in which the Pecora and Carroll method fail.

\section{4- CONCLUSION}

The proposed method for controlling and synchronizing typical simple chaotic system with only one nonlinear term can be achieved without depending on the configuration of the drive signal besides minimizing the error of synchronization Numerical results verify the validity and effectiveness of the control method. 


\section{References}

[1] W. L. Ditto and K. Showalter., "Focus issue: Control and synchronization of chaos," Chaos 7 (4)(1997).

[2] S. Boccaletti, C. Grebogi, Y.-C. Lai, and Juergen Kurth), "Focus issue: Control and Synchronization of chaotic system," Chaos 13(1)(2003).

[3] S. Boccaletti, J. Kurths, G. Osipov, D. L. Valladares, and C. Zhou, "The synchronization of chaotic systems,"' Phys. Rep. 366, 1 (2002)

[4] E. Ott, C. Grebogi, and J. A. Yorke, "Controlling a chaotic system,” Phys. Rev. Lett. 64, 1196(1990).

[5] T. L. Carroll and L. M. Pecora, “Synchronizing chaotic circuits,” IEEE Trans. CAS 38, 453 (1991).

[6] T. L. Carroll and L. M. Pecora, "Cascading synchronized chaotic systems," Physica D 67, 126- 140 (1993).

[7] L. M. Pecora and T. L. Carroll, "Driving systems with chaotic signals," Phys. Rev. A 44, 2374 (1991).

[8] T. Yamada and H. Fujisaka, "Stability theory of synchronized motion in coupled-oscillator systems. II,” Prog. Theor. Phys. 70, 1240 (1983).

[9] T. Yamada and H. Fujisaka, "Stability theory of synchronized motion in coupledoscillator systems. III,’’ Prog. Theor. Phys. 69, p32-46 (1983).

[10] E. A. Jackson and EA, Grosu I, “ An OPCL control of complex dynamic system,”Physica D 85, 1-9 (1995).

[11] E. Atlee Jackson " The OPCL control method for entrainment, model-resonance, and migration actions on multiple-attractor systems," Chaos, Vol. 7, No. 4, 1997

[12] Chen L-Q, LiuY-Z , “An OPCL approach to synchronization of chaotic and hyper chaotic map,”Int .J. of Bif. And chaos 2002, 12(5):1219-1225.

[13] R. Brown and P. Bryant, "Computing the Lyapunov spectrum of a dynamical system from an observed time series,’” Phys. Rev. A 43, 2787 (1991).

[14] M.Hasler, "transversal Lyapunov exponents and synchronization of chaotic system,” in Proc. EC-CTD'97,Budapest, 1997, pp.1211-1215

[15] ShiganChen, Zonghua Liu and Bambi Hu , "coupled synchronization of spatiotemporal chaos ,” Phys. Rev. E 59(1999), pp2817-2821.

[16] Benjamin C.Kuo, “ Automatic control systems,” prentice Hall of India, New Delhi-110001, 1982 [17]Hassan K.Khalil, “ Nonlinear systems,” Macmillan Publishing International Journal Non-LinearMechanics 42,754759,2007.

[25] Mekheimer, KH. S. and Abdelmaboud, Y.,"The influence of heat transfer and magnetic field on peristaltic transport of aNewtonian .fluid in a vertical annulus: Application ofan endoscope", Phys Letter A 372, 1657-1665,2008.

[26]Drew, D.A.,"Stability of Stokes Layer of a Dusty Gas", Physicsand Fluids 19, 2081-2084, 1979.

[27] Charm, S.E. and Kurkland, G.S.,"Blood flow and microcirculation", John Wiley Newyork, 1974.

[28] Tam. C. K. W., "The drag on a cloud of spherical particles in low Reynolds number flow", Journal Fluid Mechanics 38, 537-546, 1969. 